\title{
Instrumentation Testing for TREAT Experiments
}

\author{
Brady Francis Cameron, Austin D Fleming
}

August 2019

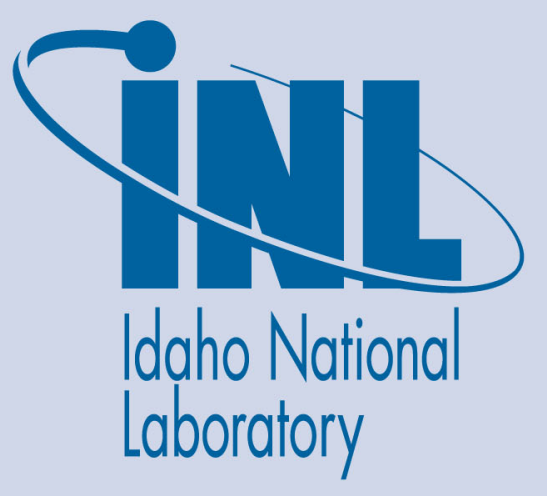

The INL is a U.S. Department of Energy National Laboratory operated by Battelle Energy Alliance 


\title{
Instrumentation Testing for TREAT Experiments
}

\author{
Brady Francis Cameron, Austin D Fleming
}

August 2019

Idaho National Laboratory Idaho Falls, Idaho 83415

http://www.inl.gov

Prepared for the

U.S. Department of Energy

Under DOE Idaho Operations Office

Contract DE-AC07-05ID14517 


\section{Instrumentation Testing for TREAT Experiments}

Brady Cameron ${ }^{1,2}$, Austin Fleming ${ }^{1}$, Heng Ban²

Idaho National Laboratory, ${ }^{2}$ Mechanical Engineering and Material Science, University of Pittsburgh

\section{Objective}

To test sensors and measurement devices prior to TREAT irradiations to ensure proper collection of data.

\section{Background}

Measurement science is crucial in order to obtain reliable data during experiments. Experimental success is dependent on:

- Accuracy \& resolution of sensor

- Understanding of time response characteristics

Separate effects understanding of sensor performance

Comprehensive uncertainty analysis

A good example of sensor limitation is poorly attached thermocouples. Inadequate contact between thermocouples and fuel cladding can cause larger thermal resistances to exist, which introduce large error in temperature measurements

Figure 1: Transient Reactor Test Facility

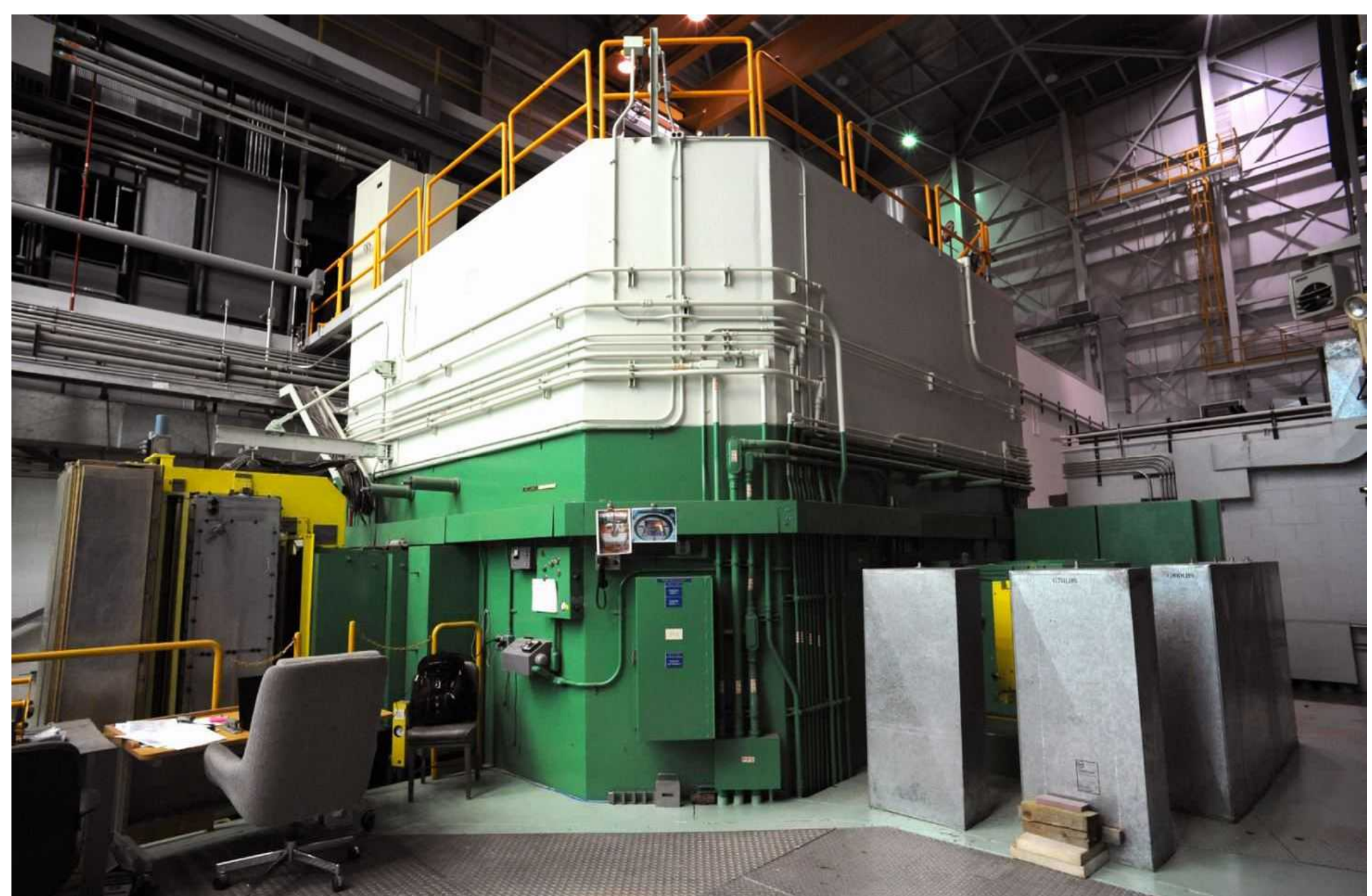

\section{Approach}

Develop mockup experiments in a laboratory environment. Benchtop mockups aid in:

- $\quad$ Better understanding of instrumentation performance

- Development of improved measurement strategies

Characterize experiment capsule and measurement performance A benchtop mockup of the Separate Effects Test Holder (SETH) capsule is made with a heater cartridge placed inside of a zircaloy-4 tube with thermocouples and pyrometers attached.
Figure 2: SETH Capsule and Type$K$ Time Response

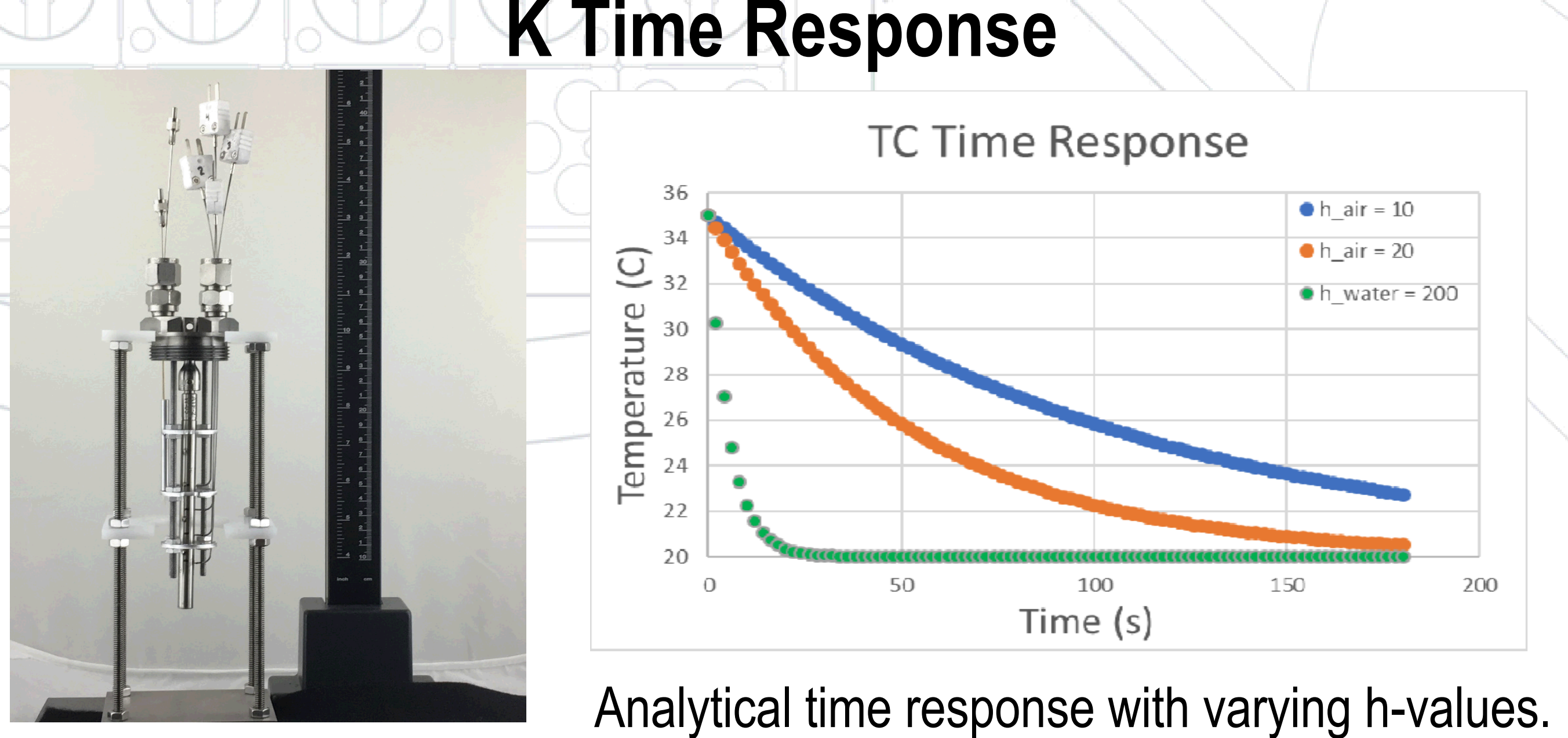

Evaluating the attachment of thermocouples is a good example of how mockup experiments can be used to evaluate sensor performance.

- Thermal resistance between thermocouple and fuel cladding results in a change in time response of the sensor

- Weldment of TCs can be effectively characterized prior to experiment.

- Sensor response can then be analyzed, in situ, prior to TREAT experiments to characterize TC contact.

\section{Theory \& Results}

Applying $500 \mathrm{~mA}$ of alternating current (To eliminate the Peltier effect) through a thermocouple will raise the temperature of the TC along the entire length ${ }^{[1]}$. Then as the thermocouples cools, the transient temperature profile at the hot junction can be recorded and the time constant can be determined. Any shift in time constant will reflect a change in weldment of the thermocouple.

Figure 3: Idealized Thermocouple a) Simple b) Effective heat c) Effective heat thermocouple generation capacity \& conductivity

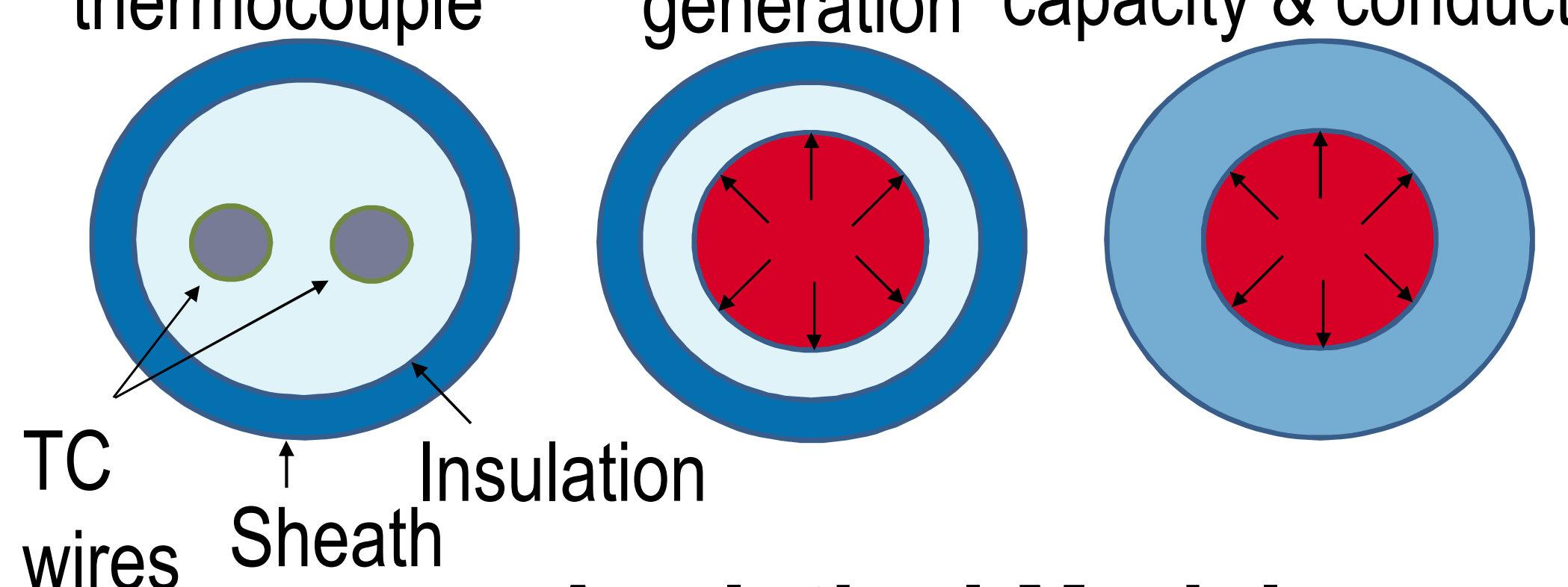

wires Sheath Analytical Model

Lumped capacitance is used to model the system, where the temperature distribution is given by:

$$
T(t)=T_{\infty}-\left(T_{i}-T_{\infty}\right) * \exp \left\{-\left(\frac{h A}{\rho V c_{p}}\right) * t\right\}
$$

And valid when

$$
B i=\frac{h V}{A k}<0.1
$$

Where, $h$ is the coefficient of convention, $A$ is the surface area, $V$ is the volume, $\rho$ is density, $c_{p}$ is specific heat, and $k$ is thermal conductivity A schematic is shown in Figure 3 where a thermocouple is modeled as a simple cylinder neglecting end effects. Superimposing isotherms yields a circular geometry that encompasses both thermocouple wires. Idealizing this center temperature distribution leads to a uniform heat generation, depicted by the red center. The effective heat compacity $(\rho \mathrm{V})$ and effective thermal conductivity $(k)$ can then be determined by transforming part $a$ and part $b$ in Figure 1 into part c, respectively.

\section{Benchtop Setup}

The benchtop setup consists of using a series of DAQ's and LabVIEW to capture data, which can be seen in Figure 4. Development of a LabVIEW program to heat thermocouples and read TC inputs using relays will be used to test weldment. A follow up program will read in multiple TC and pyromete sensors while varying a heater cartridge to act as an all-in-one instrumentation pre-check for TREAT experiments.

Figure 4: Benchtop setup and Cladding Holder
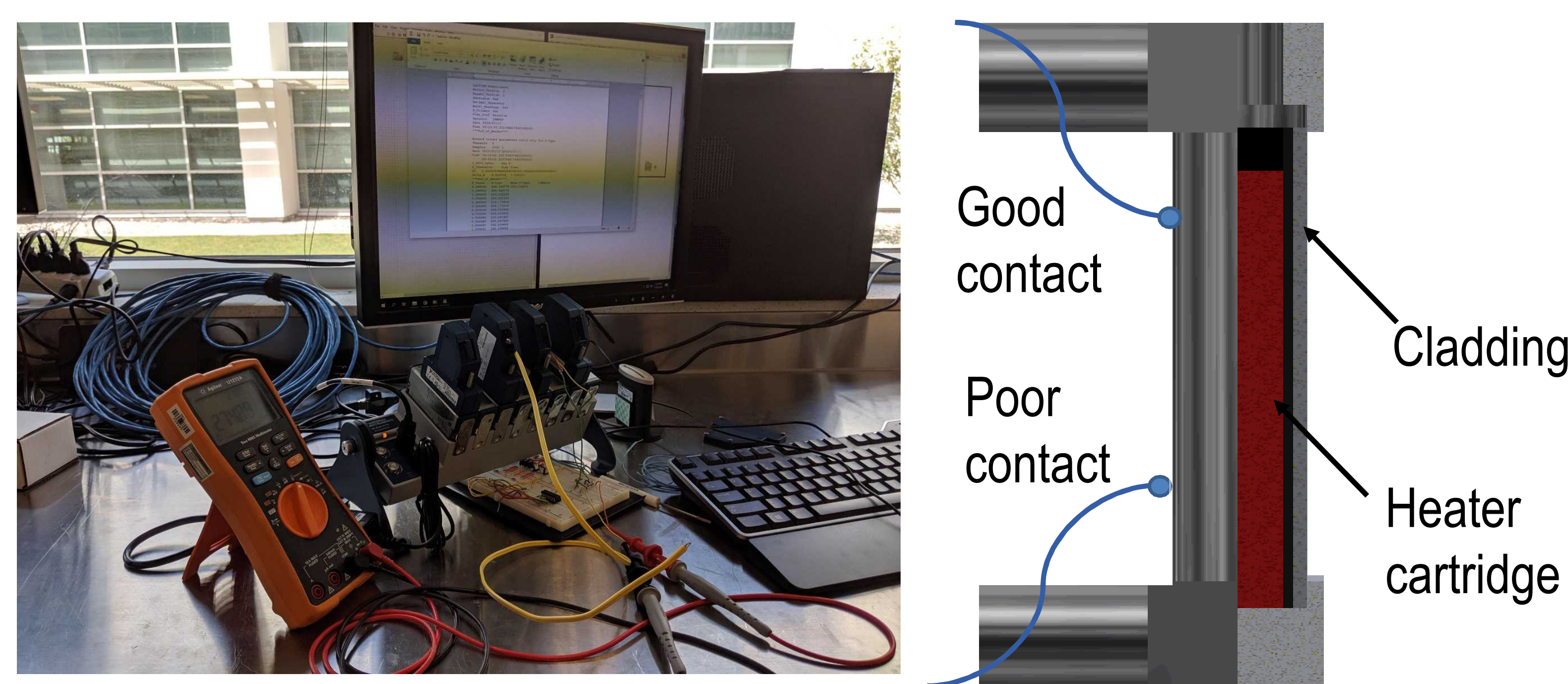

\section{Conclusion}

- Accurate temperature measurements during TREAT experiments are crucial for fundamental understanding of nuclear related phenomena.

- Preliminary testing of instrumentation prior to TREAT experiments are necessary for achieving accurate and reliable results

- Innovative techniques have been developed to characterize thermocouple attachment.

Reference:

[1] Hashemian, HM (2003) Response Time and Drift Testing, Instrument Engineers' Handbook, Fourth Edition,
Chapter 1.9m, Process Measurement and Analysis, CRC Press.

University of Pittsburgh 